\title{
Beispiel zur kompetenzorientierten Reifeprüfung in GW: Steuerpolitik in Österreich
}

paulhofmann@yahoo.de, Meinhardinum Stams \& Pädagogische Hochschule Tirol

\section{Themenbereich: Wirtschafts- und Sozialpolitik in Österreich}

\section{Problem- und Situationsbeschreibung}

„Um seine Aufgaben erfüllen zu können, braucht der Staat Einnahmen. Zu diesem Zweck erhebt er Steuern, Gebühren und sonstige Abgaben. Alle Bürgerinnen und Bürger haben die Pflicht, im Rahmen der vom Parlament beschlossenen Gesetze ihren Anteil zur Finanzierung öffentlicher Leistungen beizutragen." (BMF 2015).

\section{Arbeitsaufgaben}

1. Führen Sie die wichtigsten Steuern in Österreich an und geben Sie dazu jeweils eine kurze Erklärung (Anforderungsbereich I).

2. $\mathrm{Ab}$ dem 01.01.2016 gibt es in Österreich neue Lohnsteuertarife. Vergleichen Sie diese mit den bis dahin geltenden Tarifen (siehe M1) und geben Sie mögliche Auswirkungen für die Steuerzahler und den Staat an (Anforderungsbereich II).

3. Manche Politiker/innen befürworten die Einführung einer Flat Tax. Interpretieren Sie dazu die Karikatur M2 (Anforderungsbereich II). Nehmen Sie zu diesem Steuermodell über die Karikatur hinaus kritisch Stellung (Anforderungsbereich III).

4. In Österreich wurde 2008 die Erbschafts- und Schenkungssteuer abgeschafft. Im Zuge der Finanzierung der Steuerreform 2016 wurde die Wiedereinführung dieser Steuer diskutiert. Bewerten Sie unter Verwendung der Materialien M3 und M4 eine zukünftig mögliche Wiedereinführung.

\section{Erwartungshorizont}

1. Die Kandidatin/der Kandidat sollte folgende Kategorien anführen und kurz erklären: Lohn- bzw. Einkommenssteuer, Umsatz- bzw. Mehrwertsteu- er, Körperschaftssteuer, Grundsteuer, Wertpapiersteuer, KESt, Mineralölsteuer, Tabaksteuer, KFZ-Steuer

2. Die Kandidatin/der Kandidat sollte zur Lösung der Aufgabe das bis 01.01.2016 geltende Steuertarifsystem kennen. Muss es aber nicht ausführlich anführen, sondern die neue Stufeneinteilung (geltend ab 01.01.2016) herausarbeiten und mögliche Auswirkungen davon (z. B. Entlastungen, wer wird belastet, wer wird entlastet?).

3. Die Kandidatin/der Kandidat soll sich kritisch mit einem System auseinandersetzen, das nur eine Steuerstufe (z. B. 25 \%) für alle vorsieht und mögliche Auswirkungen auf unterschiedliche Steuerzahler/innen sowie auf das Finanzverwaltungssystem herausarbeiten.

4. Die Kandidatin/ der Kandidat soll dazu eine eigene fundierte Meinung mit Pro- und ContraArgumenten vertreten.

\section{$4 \quad$ Material}

M1 Die neuen Lohnsteuertarife ab 2016 (Abb. 1)

M2 Karikatur zu Flat Tax (Abb. 2)

M3 Pro-Argumentation zu Schenkungs- bzw. Erbschaftssteuer (Textbox 1)

M4 Einige Contra-Argumentationen zu Schenkungsbzw. Erbschaftssteuer (Textbox 2)

\section{Literaturverzeichnis}

BMF - Bundesministerium für Finanzen (2015): Steuern. https://www.bmf.gv.at/steuern/startseite-steuern.html (17.01.2016).

Humer, S. (2014): Aufkommen von Erbschaftssteuern. Modellrechnung exemplarischer Tarife. In: Wirtschaft und Gesellschaft 40 (1), 151-160.

Neumayer, C. (2012): Das Für und Wider zu Erbschafts- und Schenkungssteuer. Beitrag im Blog der Industriellenvereinigung vom 23.08.2012. http://www. christoph-neumayer.at/das-fur-und-wider-zu-erbschaftsund-schenkungssteuer/ (17.01.2016). 


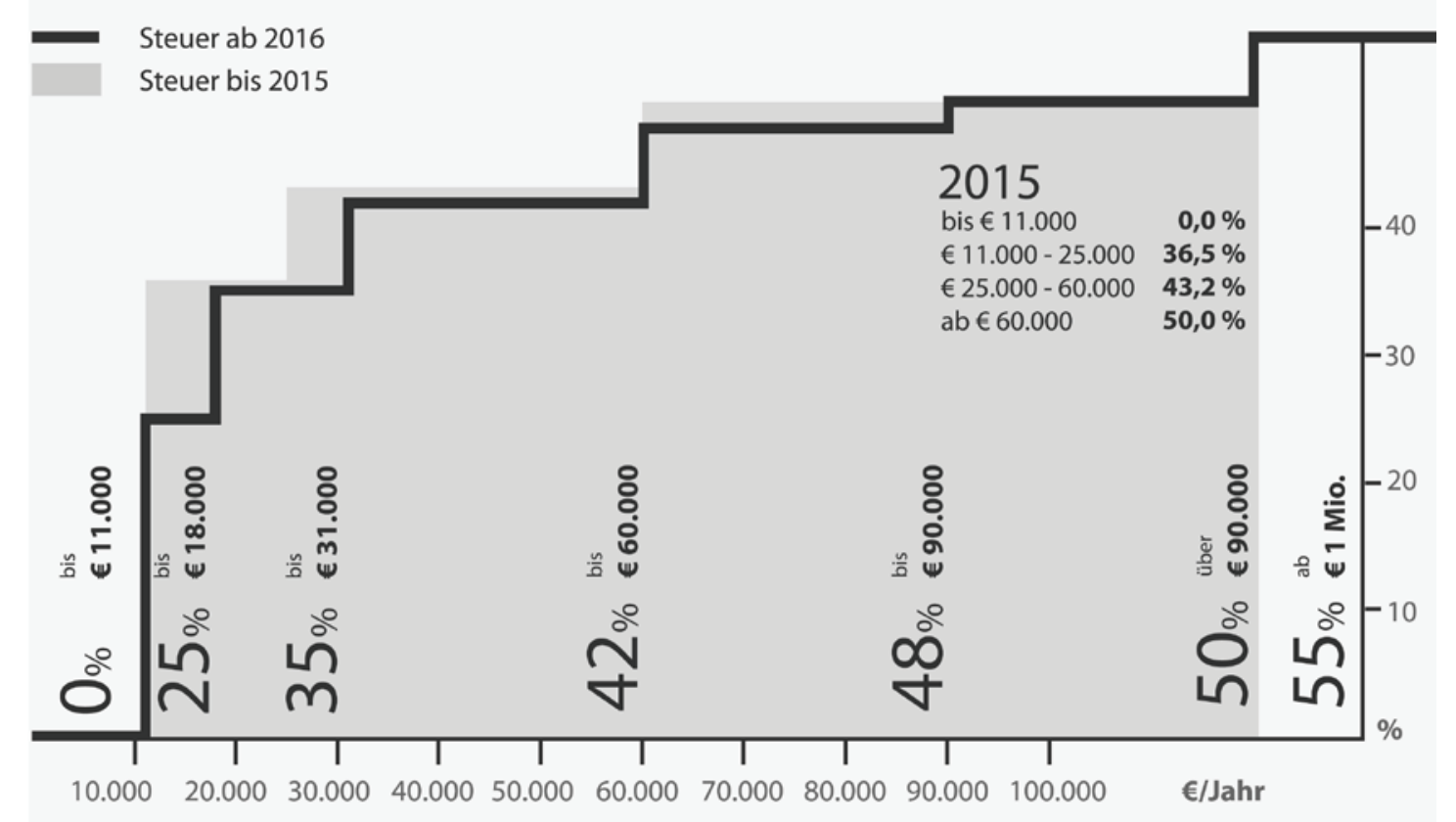

Abb. 1: Die neuen Lohnsteuerstufen nach Jahreseinkommen ab 2016 (Grafik: Thomas Egger, nach BMF 2015, https://www.help.gv.at/, Abruf am 06.02.2016)

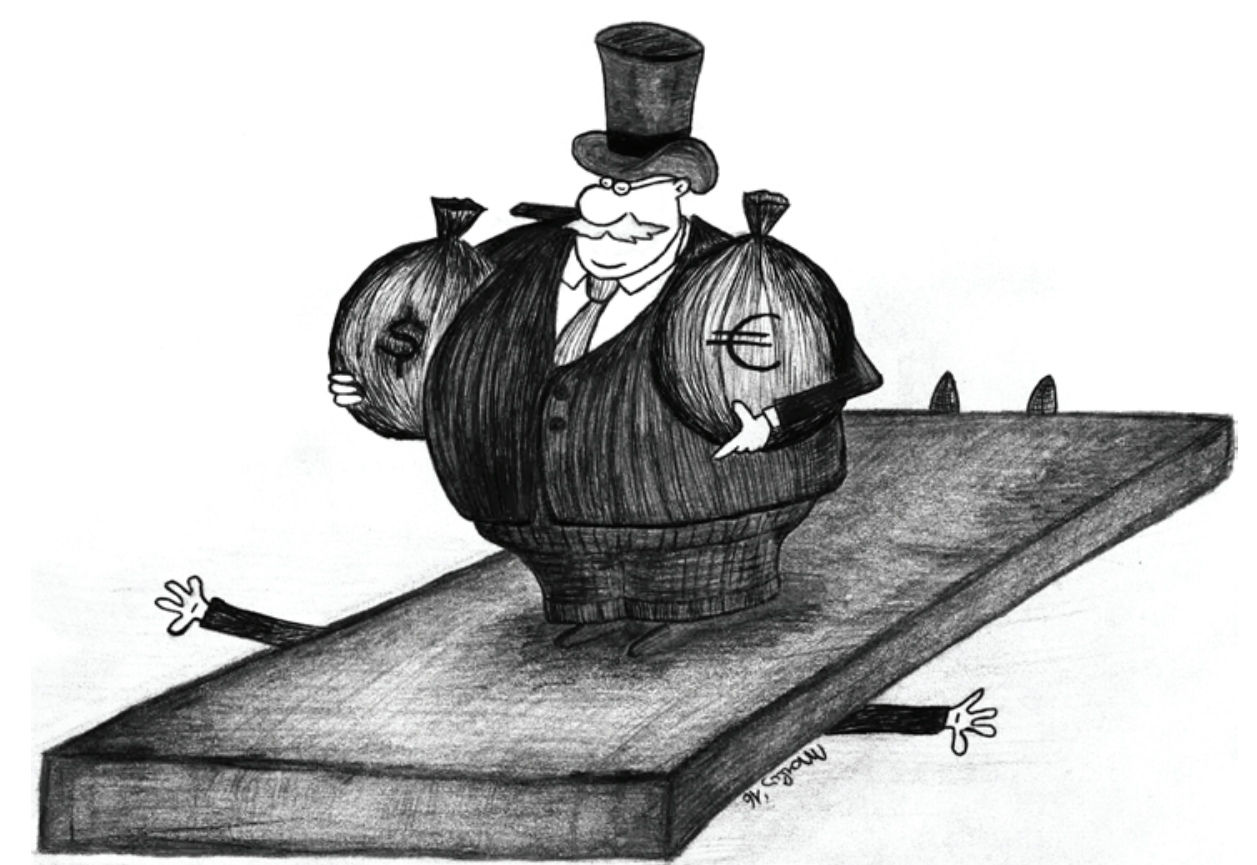

Abb. 2: Karikatur „How the flat tax works" (Grafik von Marlis Pardeller nach der Idee von edstein 'II, edsteinink.com)

\begin{abstract}
Vermögen sind in Österreich ungleich verteilt. Mit einem Freibetrag von $€ 500000$ würden im Durchschnitt die unteren $95 \%$ aller Vermögensübertragungen von der Erbschaftssteuer befreit, dieser Anteil stiege mit einem Freibetrag von $€ 1000000$ auf $98 \%$. Eine Verknüpfung solch relativ großzügiger Freibeträge mit der Bemessung der Vermögen an ihren Verkehrswerten ermöglicht es, einen Großteil der Vermögensübertragungen nicht oder nur in einem geringen Ausmaß zu belasten und gleichzeitig doch ein substanzielles Aufkommen von über einer halben Mrd. Euro pro Jahr zu generieren. Im Zusammenhang mit der angespannten Lage der öffentlichen Haushalte ergäbe sich somit der notwendige Spielraum, um eine Umschichtung der steuerlichen Belastung von Arbeit und Vermögen zu ermöglichen. Eine solche Maßnahme sollte positive Wachstumseffekte nach sich ziehen (Humer 2014, 151 ff.).
\end{abstract}


- Eine Erbschafts- und Schenkungssteuer mit einem Freibetrag von einer Million Euro (wie es die SPÖ derzeit andenkt), würde laut Erbschafts- und Schenkungssteuerbescheiden aus dem Jahr 2007 (letzte verfügbare ganzjährige Statistik vor dem Auslaufen der Erbschafts- und Schenkungssteuer im Jahr 2008) nach alter Rechtslage ganze 49 Steuerfälle mit einem Gesamtaufkommen von 11,2 Mio. Euro auslösen. Das würde kaum den dafür entstehenden Verwaltungsaufwand rechtfertigen.

- Der Grad der Erfassung des Vermögens ist lückenhaft: Gold, Schmuck, Münzen, Antiquitäten, Kunstwerke sind kaum erfassbar, es drohen Verwaltungsaufwand und „Schnüffelei“.

- Eine Besteuerung von Betriebsvermögen bzw. Unternehmensbeteiligungen ist fatal, besonders bei Familienunternehmen (Neumayer 2012). 